\title{
Enhanced Industrial Maintenance Work Task Planning by Using Virtual Engineering Tools and Haptic User Interfaces
}

\author{
Simo-Pekka Leino ${ }^{1}$, Salla Lind ${ }^{1}$, Matthieu Poyade ${ }^{2}$, Sauli Kiviranta ${ }^{1}$, \\ Petteri Multanen ${ }^{3}$, Arcadio Reyes-Lecuona ${ }^{2}$, Ari Mäkiranta ${ }^{3}$, and Ali Muhammad ${ }^{3}$ \\ ${ }^{1}$ VTT Technical Research Centre of Finland, Tekniikankatu 1, 33580, Finland \\ ${ }^{2}$ Departamento de Tecnología Electrónica, ETSI de Telecomunicación, \\ Universidad de Málaga, Campus de Teatinos, s/n, 29071 Málaga, Spain \\ ${ }^{3}$ Tampere University of Technology, IHA Department of Intelligent Hydraulics and \\ Automation, Finland \\ \{simo-pekka.leino, salla.lind, sauli.kiviranta\}@vtt.fi, \\ \{matthieu.poyade, areyes\} @uma.es, \\ \{petteri.multanen, ari.makiranta, muhammad.ali\}@tut.fi
}

\begin{abstract}
Good maintainability is an essential feature for machines and processes in industry. It promotes, among others, maintenance safety, postmaintenance reliability and cost-effective maintenance by ensuring quick and easy operation and short downtime. Virtual engineering tools provide an effective way for maintainability design already during the design phase. Machine designers may not consider maintenance tasks systematically, which can leave important task details open. The missing detail planning can contribute significantly to the probability of safety or reliability risks. So far, generic tools or facilities for planning demanding maintenance tasks in detail have not been available for companies' independent use. Another challenge is to develop and apply better user interfaces for design processes. Virtual engineering tools, such as virtual reality (VR) and haptics, provide a potential solution for improving maintenance planning and maintainability design. This paper introduces development and benefits of a new haptic interface for planning and training industrial maintenance tasks. The paper introduces a test with haptics tools in virtual maintenance case examples. As a conclusion we will sum up, whether the use of a haptic user interface would enhance task planning and maintainability design. In addition, we propose a set of recommendations regarding use of haptics in maintenance planning and maintainability design.
\end{abstract}

Keywords: Haptics, Virtual Environments, Maintenance.

\section{Introduction}

After-sales service is nowadays a focal part of business for machine manufacturers. For their customers, downtime of industrial plants is very expensive, which reflects as requirements for availability and effectiveness of maintenance services. Good maintainability is an essential feature for machines and processes in industry. It promotes, among others, maintenance safety, post-maintenance reliability and cost-effective 
maintenance by ensuring quick and easy operation and short downtime. Maintainability is also an inherent part of PLM, which emphasizes the users' viewpoint throughout the product lifecycle. [1]

Effectiveness of maintenance can be promoted with good maintenance task planning. However, generic tools or facilities for identifying and planning demanding maintenance tasks have not been available for companies' independent use. Another challenge is to develop and adopt better user interfaces for design processes. So far, design engineers have paid attention on higher level to maintenance tasks, leaving the task details open. The missing detail planning can contribute to the probability of safety or reliability risks [2]. As the current virtual engineering tools are lacking natural user interfaces, identification of the challenges of manual work tasks and circumstances is challenging using a CAD program in a desktop computer [3]. A potential solution for improving maintenance planning and maintainability design is using virtual engineering tools, such as virtual environments (VE) and haptics. However, the key challenge has been integrating such tools into the companies' production design processes.

In order to improve maintainability design and maintenance task planning, a research project called Virvo [2] was launched in 2006. In the research project we had two industrial case studies: maintenance of a heavy duty rock crusher and modernization of an elevator. The Virvo project has two main aims: 1) to develop a method for planning of critical maintenance work tasks in industry, and 2) to integrate the method to be a functional part of engineering processes.

This paper introduces development and implementation of a new haptic interface for maintenance task planning. The paper focuses on test of a haptic tool in virtual maintenance case examples. In these cases, a haptics tool was added to VE models with aim to find out if and how the use of haptics can produce information for maintainability design and maintenance task planning. As the maintenance test case had to be specific and independent from the confidential industrial case studies, we decided to make the test using a fictitious case on car maintenance. The relevant findings discussed with aim to consider their application in planning maintenance tasks in VE's. As a conclusion we will sum up, whether the use of a haptic user interface would enhance task planning and maintainability design. In addition, we propose a set of recommendations regarding use of haptics in maintenance planning and maintainability design.

\section{Technical Background}

\subsection{Virtual Environments and Maintainability}

Virtual Environment (VE) can be defined as a plausible artificial environment, which is created by technical means and allows interaction between the user and the environment [4]. Typically CAD-software enables one-way interaction, i.e. it reacts to commands given by the user, but does not provide real feedback to the user. Virtual environment system can consist of software, virtual models (CAD-models, digital 
humans, etc.), data communication, computers, display devices (monitors, immersive CAVEs), user interface devices, other devices and their drivers. The appropriate installation depends on the application.

The projection of a virtual model helps the users to understand the content of the design concept more reliably when compared to drawings and desktop display. Depending on the application the impressiveness of presentation can further emphasized by utilizing stereoscopic image, multiple projection or immersive virtual realities.

Currently only the most critical work tasks can be analyzed using VE tools, which can be costly or time-consuming to use. Thus, the tasks must be prioritized due to their criticality. Such tasks can be, for example, complex tasks or tasks involving high safety risks.

Maintainability can be defined quantitatively as the probability of performing a successful repair action within a given time [5]. Thus, maintainability can be defined quantitatively with equation (1), where $\mu$ is repair rate.

$$
M(t)=1-e^{(\mu * t)} .
$$

Following this definition, the qualitative maintainability features are those promoting ease and speed of maintenance task [5]. From the worker's viewpoint, system maintainability includes, among others, features affecting on ergonomics during the task execution. Certain maintainability indexes include in the concept of maintainability measurable variables, such as number of tasks to complete the operation, required time and number of tools. In addition, such indexes pay attention also to a system's qualitative features, such as accessibility and reachability. (see e.g. [6] [7]) The maintainability indexes aim to give maintainability a quantitative value based on qualitative and quantitative system and task features. The maintainability features can be assessed using virtual engineering tools. In addition, maintenance task details can be identified and analyzed in virtual environments already in the system design phase. The use of virtual technologies is of special interest in large-scale projects where the design and planning of maintenance devices and operations is heavily relied on virtual prototypes, due to the high costs, large size and complexity of physical prototypes. As an example, virtual models have been used extensively during the development of teleportation system for the maintenance of ITER divertor [8].

\subsection{Haptics}

The word 'haptics' refers to the sense of touch. This encompasses both tactile and kinesthetic sensory information [9], which suppose two different philosophies in touching and perceiving surrounded objects within an environment.

The use of haptic interfaces is becoming more typical. The haptic devices are small manipulators with several degrees of freedom with the capability of force reflection to the operator's hand (see e.g. [10]). The information about the operation is received from the virtual environment as sense of forces. Nowadays, several haptic devices are available in the market, providing a variety of workspaces and force feedback options. Irrespective their complexity, they all have force reflecting mechanisms. With controls and software tools, these devices can be applied in various ways to meet 
different user needs and requirements. The desired dynamics of the device can be modified in terms of mass, viscosity and stiffness to simulate the impedance matching with the maintenance task.

Applied to VEs, haptics results in the reciprocal communication between human and machine sensory motor channels [9], inducing force feedback enhancing strongly the tri-dimensional interaction in VEs [9] [11]. Furthermore, combined with VEs, haptic devices can provide new possibilities for the machine manufacturers. The manufacturers can interact with the product during the design phase and examine the associated maintenance procedures. This enables early improvement of maintainability through modification and possible redesign. For the customer, utilization of haptic devices and VEs helps planning, practicing and simulating maintenance task in the virtual environment before executing the task with the real product. In this way, a great number of errors and unpredictable situations can be identified and avoided before performing the actual maintenance task.

When implementing haptics in VEs, different software solutions are needed to integrate the required hardware. Usually, haptic interface manufacturers provide their own Application Programming Interface (API), which includes a set of functions in order to facilitate implementation onto VEs graphical engine. For example, Sensable Technologies provides the OpenHaptics ${ }^{\circledR}$ toolkit 2.0 [12], a free API oriented to OpenGL programming for Sensable Phantom ${ }^{\circledR}$ devices [13].

\section{Methods}

The paper bases on test of haptics tools in virtual maintenance case examples. Within these cases, a haptics interface, combined with VE models, was applied in maintenance planning process with aim to find out if and how the use of haptics can produce information for maintainability design and maintenance task planning. A driver was programmed in order to connect a haptic device with VE software. The test was carried out using a industrial rock crusher maintenance case and a fictitious car maintenance case.

First, we developed the necessary VE models. The development was carried out using Dassault/Virtools simulation software. We also described the data processing from CAD models to hapticalized virtual environment model. Second, we built a software interface (driver) between a commercial engineering tool (Dassault/Virtools) and a haptics device (SensAble Phantom ${ }^{\circledR}$ ). Third, we created VE models for connecting VE with the haptic user interface for product development and maintenance planning processes. Fourth, we conducted a limited user test with 11 persons in order to explore usability and added value of haptic user interface in maintenance operations. The user tests consisted of three different test cases: 1) manipulation of 3D-parts in VE, 2) welding task of car maintenance, 3) re-assembly task of rock crusher maintenance. The most extensive tests were executed with the rock crusher. In that case, the actual test case is confidential, whereas the results regarding the use and feasibility of haptics are public. 


\subsection{Usability Test: Setting}

In the welding task, test persons connected two parts of a rock crusher during reassembly task of crusher maintenance. The virtual model was built from CAD models, which were imported to Virtools simulation software. Haptic interface was built using the created haptics driver between Virtools and SensAble Phantom ${ }^{\circledR}$ device. The group of 11 test persons included: development engineer (3), project manager (2), machine engineer (2), mechanics engineer, mechanic, documentation engineer, design engineer. All of the test persons were employees of Metso Minerals company. During the test, they had the possibility to try a general haptics demo before the actual usability query. Each test person executed the welding task. After that, they filled a questionnaire form with ten statements. In addition to the statements, the questionnaire charted background information regarding persons' experience level (no experience, little experience, professional) with the different design tools and applications (3D models, CAD, VEs, simulation, haptics).

The questionnaire included the following ten statements: 1) haptic interface is easy to use, 2) user interface is natural, 3) haptics improves 6DOF navigation, 4) haptic device easier to use than 3D-mouse, 5) sense feedback helps me, 6) force feedback helps me, 7) haptics improves design work, 8) haptics improves training of work tasks, 9) I could adopt haptics as working tool, 10) overall attitude to haptics is positive. The statements had two answer options, i.e. yes and no. In addition, the test persons had also the possibility to give their free comments about haptics and ideas about haptics applications in industry.

\subsection{Applied Software and Devices}

The studies involved several tools and applications. Virtools ${ }^{\mathrm{TM}}$ is a comprehensive platform for creating highly interactive 3D applications. Programming is based on separation of objects, data and behaviors with intuitive user interface with real-time visualization window and graphical programming. With Virtools ${ }^{\mathrm{TM}}$ Scripting Language or the Virtools C++ SDK it is possible to implement customized functions and custom devices. (see: www.virtools.com)

Phantom Omni ${ }^{\circledR}$ and Desktop ${ }^{\circledR}$ interfaces are affordable electromechanical kinesthetic haptic desktop devices, manufactured by SensAble Technologies Inc ${ }^{\circledR}$. The Phantom system provides a force reflecting interface between users and computers. It generates a pleasurable tri-dimensional haptic interaction with VEs due to the high degree of maneuverability. Depending on the configuration, the Phantom-based interaction can be realized through a stylus grip that users can comfortably handle as a pen, or a fingertip thimble [13].

The Phantom system tracks users' force and motion information to the 6 DOF of maneuverability. In turn, the Phantom system provides feedback to $3 \mathrm{DOF}$, high performance force effects [13], which maximum can reach $3.3 \mathrm{~N}$ for the Phantom Omni ${ }^{\circledR}$ and $7.9 \mathrm{~N}$ for the Phantom Desktop ${ }^{\circledR}$.

Open-Haptics ${ }^{\circledR}$ toolkit 2.0 is a two layer haptic library. Higher level library, HLAPI, provides advanced support to haptic rendering, managed into a threading model. Haptic display, collision detection and Force Feedback are accomplished onto three separate threads that respectively update at 30, 100 and $1 \mathrm{KHz}$. OpenHaptics ${ }^{\circledR}$ 
toolkit 2.0 provides a set of pre-written functions that allows setting up and combining various custom force effects, such as stiffness, damping, friction, dynamic friction and viscosity.

\section{Results}

The developed haptic interface enables the use of SensAble Phantom ${ }^{\circledR}$ haptic device with commercial Virtools VE development platform. The developed user interface for maintenance planning is integrated into a common product process of industrial companies (Fig. 1).

The product process includes following phases: concept design, detail design and engineering, prototyping and testing, production, operation and maintenance of products. Haptic interface is integrated into this process so, that $3 \mathrm{D}$-models (CAD) generated in concept design and detail design (engineering) phases are exported into Virtual Environment. CAD-models are converted into proper file format of Virtools simulation software and simplified in order to reduce complexity of the model. Some additional models can be combined to VE, if needed.

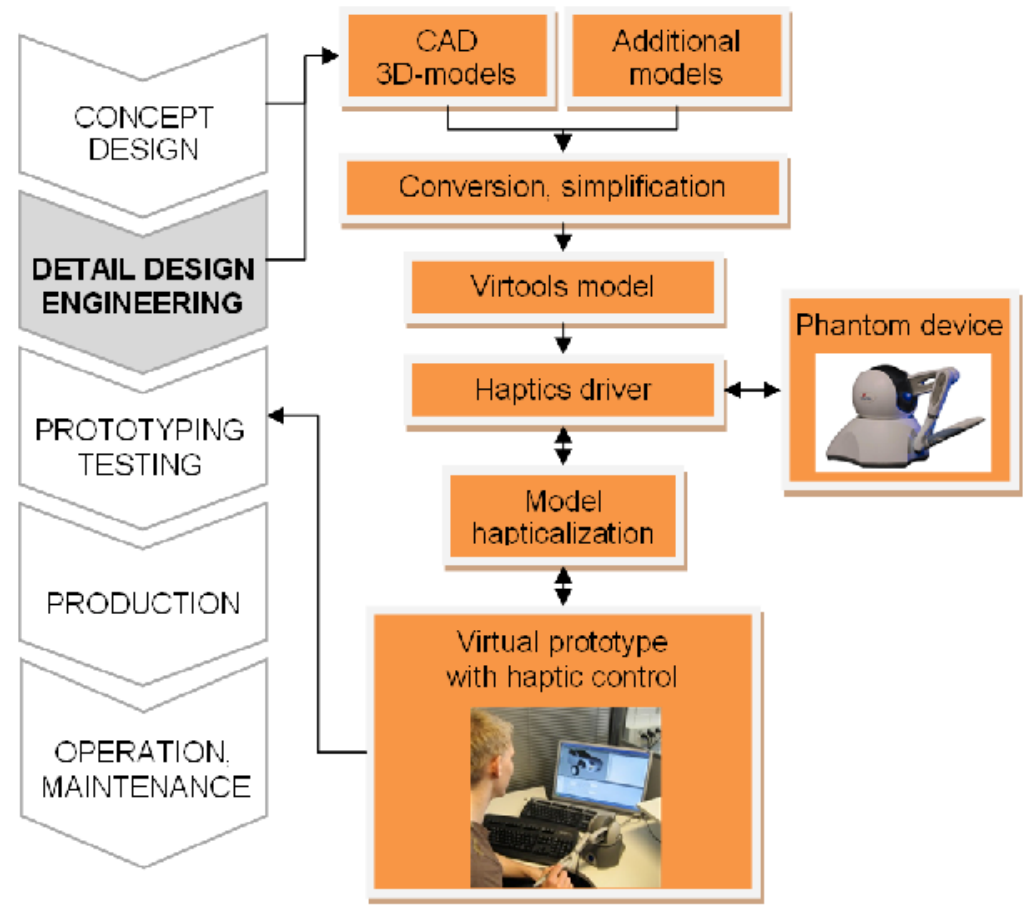

Fig. 1. Haptic user interface of maintenance planning integrated into a product process 
Haptics driver connects SensAble Phantom ${ }^{\circledR}$ haptic device and Virtools. The haptic interface (driver) is introduced in more details in [14]. The driver also takes care of hapticalization of virtual models. The driver can be configured via graphical building block inside Virtools software. Engineers can plan and assess maintenance operations with haptics in Virtual Environment and give feedback into product process during prototyping phase.

The case studies explored the usability of the created methodology and haptic interface. Researchers and engineers of the partaking company assessed the related benefits. Haptic user interface was assessed to be easy to use and it has natural user interface. Following enhancements were reported during the user tests: Haptic interface improves 6 DOF navigation in a VE, haptic interface is easier to use than 3D-mouse, sense feedback of VE is moderate and force feedback is good. Half of the respondents considered haptics as a potential improvement in their design work, while all respondents found haptics as an improvement in industrial work task training. Half of the respondents considered it possible to adopt haptic interface as a work tool. Results from engineer user test are reported with more details in [14].

Haptic device has limitations on capability to process high accuracy models. However, while direct CAD models from industrial partners have been too heavy to process for the computers, there are now good possibilities to reduce model details in such a way that the haptic devices can be applies. Overall stability of the software has been very good. Interface of the haptic driver is also very easy to use.

\section{Discussion and Conclusions}

This paper introduces a new haptic user interface, which was applied to virtual maintenance planning, and integrated to product development process. Compatibility with product development processes and commercial engineering software tools can promote fluent implementation and utilization of the new haptic interface in industrial companies. Nowadays relatively low-cost and easy-to-use haptics devices enable cost-effective usage of haptics in maintenance engineering. Considering the relation between performance and cost efficiency, the introduced combination of device and software can be a good option for the development of inexpensive Virtual Reality applications including haptic tri-dimensional interaction.

The case studies indicate that the developed haptic interface enhances maintenance task planning and maintainability design as the interface is easy to use and it enables more natural user interface. Users can sense, for instance, the surfaces, mass and inertia feelings of the assembly parts in the maintenance work task. The interface provides also a six degree of freedom navigation in virtual environment and control of the disassembled/assembled parts. Furthermore, with haptics interface the design engineers can better identify difficulties and review the feasibility of maintenance work tasks. For example, force feedback could give a hint about mass of big machine parts. Training with haptics seems to be very promising application in industrial maintenance and assembly applications. However, adopting haptics as a design tool is up to the person.

During the studies, we identified some future improvements for the haptic interface. One technical drawback of the current driver was lack of capability to give 
feedback between manipulated object and environment. Furthermore, navigation inside VE may be challenging for the new users. Solutions for overcoming this problem include, for example, adding certain camera movement algorithms to improve sense of location. Best solution for this would be tracking for user's head motions, which would bring depth sensing to state of human's capability for sensing spatial locations in reality.

For maintenance planning and maintainability design, detailed planning using virtual engineering tools provide new possibilities to explore system features at an early stage. Such tools can help to assess, for example, reachability and accessibility to the maintenance area. They also help to estimate the complexity and feasibility of various maintenance tasks. In future applications, different kinds of haptics tools and VEs could also make it possible to identify and assess the task-related risks and complexity of the work phases during disassembly and reassembly. Moreover, haptics and VEs can have significant benefits in maintenance training and planning, as they provide a safe and realistic way to examine the tasks. However, some detailed studies are required to find the optimal solutions for generic industrial applications.

In future, the development should focus on collision feedback between manipulated object and the surrounding environment, which is essential in order to make assembly applications realistic. Building training simulators, requiring feedback only from the object surfaces, is probably more potential application than designing big machines in industry. With current virtual reality development tools, it is relatively easy to gather user performance data from such training simulators. Post processing this data for performance evaluation can be easily done in different software or can be shown in real time for the users. Sense and force feedback can be seen as good addition to VEs, but increased realism in haptics interfaces and enhanced exploitation of haptics in VEs still need development.

\section{References}

1. Lind, S., Leino, S.-P., Multanen, P., Mäkiranta, A., Heikkilä, J.: A virtual engineering based method for maintainability design. In: 4th International Conference on Maintenance and Facility Management, Rome, Italy, April 22-24 (2009)

2. Leino, S.-P., Helin, K., Lind, S., Viitaniemi, J., Multanen, P., Mäkiranta, A., Lahtinen, J., Nuutinen, P., Heikkilä, J., Martikainen, T.: Virtual engineering and remote operation in design, training and completion of demanding maintenance work tasks in challenging industrial plants (Virvo). In: MaSi Programme 2005-2009. Yearbook 2008. Tekes (2008)

3. Kimura, F., Yamane, N.: Haptic Environment for Designing Human Interface of Virtual Mechanical Products. The International Academy for Production Engineering, CIRP Annals - Manufacturing Technology 55, 127-130 (2006)

4. Reitmaa, I., Vanhala, J., Kauttu, A., Antila, M.: Virtuaaliympäristöt - kuvan sisälle vievät tekniikat. 2nd edn. TEKES, Helsinki (1996)

5. Sharma, R.K., Kumar, S.: Performance Modeling in Critical Systems Using RAM Analysis. Reliability Engineering and System Safety 93, 913-919 (2008)

6. SAE J817-2. Engineering Design Serviceability Guidelines - Construction and Industrial Machinery - Maintainability Index - Off-Road Work Machines. Surface Vehicle Information Report. Society of Automotive Engineers. Warrendale, PA (March 1991) 
7. Wani, M.F., Gandhi, O.P.: Development of Maintainability Index for Mechanical Systems. Reliability Engineering and System Safety 65, 259-270 (1999)

8. Muhammad, A., Esque, S., Tolonen, M., Mattila, J., Nieminen, P., Linna, O., Vilenius, P.: Water hydraulic based teleoperation system for ITER. In: Tenth Scandinavian International Conference on Fluid Power, SICFP 2007, Tampere, Finland (2007)

9. Srinivasan, M.A., Basdogan, C.: Haptics in Virtual Environments: Taxonomy, Research Status and Challenges. Computer \& Graphics 21, 393-404 (1997)

10. Sensable Technologies, http: / /www.sensable.com/haptic-phantom-omni.htm

11. MacLean, K.E., Hayward, V.: Do It yourself Haptics: Part II. IEEE Robotics \& Automation Magazine, 104-119 (2008)

12. Itkowitz, B., Handley, J., Zhu, W.: The OpenHaptics ${ }^{\mathrm{TM}}$ Toolkit: A Library for Adding 3D Touch $^{\mathrm{TM}}$ Navigation and Haptics to Graphics Applications. In: Proc. 1st Joint Eurohaptics Conf. and Symp. Haptic Interfaces for Virtual Environment and Teleoperator Systems, World Haptics, pp. 590-591. IEEE CS Press, Pisa (2005)

13. Potts, A.: Phantom-based haptic interaction. In: Proc. Comput. Sci. discipline semin. conf. CSCI 3901 (2000),

http://mrs.umn.edu/ lopezdr/seminar/spring2000/potts.pdf

14. Poyade, M., Reyes Lecuona, A., Leino, S.-P., Kiviranta, S., Viciana Abad, R., Lind, S.: A High-Level Haptic Interface for Enhanced Interaction within Virtools ${ }^{\mathrm{TM}}$. In: 13th International Conference on Human-Computer Interaction, San Diego, USA, July 19-24 (2009) 